\title{
Tres semanas de docencia virtual en la Universidad de Murcia (II): Fisioterapia, Odontología, Cirugía y Fisiología.
}

\section{Three weeks of online teaching in the University of Murcia (II): Physiotherapy, Odontology, Surgery and Physiology.}

\author{
Gómez-Conesa $A^{\mathbf{1}}$, Ortiz, $\mathbf{A J}^{2}$, Cascales $\mathbf{P}^{3}$, García-Estañ $\mathbf{J}^{\mathbf{4}}$ \\ ${ }^{1}$ Departamento de Fisioterapia, agomez@um.es \\ ${ }^{2}$ Departamento de Estomatología, ajortiz@um.es \\ ${ }^{3}$ Departamento de Cirugía, cascalescirugia@gmail.com \\ ${ }^{4}$ Departamento de Fisiología, jgestan@um.es
}

Recibido: 27 de abril de 2020; Aceptado: 18 de mayo de 2020; Publicado: 18 de mayo de 2020

Resumen: Presentamos un resumen de las actividades que algunos de los profesores de la Facultad de Medicina de Murcia han llevado a cabo durante las 3 semanas previas a las vacaciones de primavera. En éstas, la docencia presencial tuvo que ser sustituída por actividades en línea o virtuales, a causa de la implantación del estado de alarma en España, que motivó el cierre completo de las Universidades desde el 13 de marzo de 2020. Las experiencias son de Fisioterapia, Odontología Pediátrica, Fundamentos de Cirugía y Fisiología.

Palabras clave: Docencia Virtual; Videoconferencia; Chat; Fisioterapia; Medicina; Fisiología, Cirugía, Odontología

\begin{abstract}
We present a summary of the activities that some of the professors of the Faculty of Medicine of Murcia have carried out during the 3 weeks prior to spring break. In these, face-to-face teaching had to be replaced by online or virtual activities, due to the implementation of the state of alarm in Spain, which led to the complete closure of the Universities since March 13, 2020. The experiences are from Physiotherapy, Pediatric Odontology, Surgery Basics and Physiology.
\end{abstract}

Keywords: Virtual Teaching; Videoconference; Chat; Physiotherapy; Medicine, Dentistry; Physiology, Surgery

\section{Introducción}

A partir del 13 de marzo, con la declaración del Estado de Alarma en España, todas las actividades presenciales en la Universidad cesaron bruscamente y obligaron a los profesores a pasar de forma inmediata a un sistema de docencia en línea (online) con el que seguir impartiendo docencia a sus alumnos. En este artículo presentamos algunas de las experiencias que se han llevado a cabo desde ese día hasta el viernes anterior a las vacaciones de primavera (3 de abril) en la Facultad de Medicina de la Universidad de Murcia.

Este es el segundo artículo de esta serie y en este presentamos las actividades realizadas en un par de asignaturas de Fisioterapia (Grado y Máster), en una del Grado en Odontología, Clínica Integrada de Odontología Pediátrica, y en Fundamentos de Cirugía y Fisiología del Grado en Medicina. 


\section{Grado y Máster en Fisioterapia, Profa. Antonia Gómez Conesa.}

Las asignaturas son, en el Grado de Fisioterapia, Fisioterapia de la Espalda y los Tegumentos y en el Máster de Fisioterapia Neurológica del Niño y el Adulto, Intervención Cognitiva y Funcional en Demencias.

Antes incluso de la puesta en marcha del plan de contingencia para la educación online por parte de la UMU, se tuvo que dar respuesta a las necesidades de los estudiantes de Máster (Master en Fisioterapia Neurológica del niño y el adulto) y de Grado (Fisioterapia), para dar continuidad a las actividades docentes de manera no presencial; tomar decisiones ad hoc respecto a las Prácticas Clínicas que llevaban a cabo los estudiantes en centros que iban paulatinamente suspendiendo la estancia, hasta que llegó la suspensión total por parte de la UMU; gestionar la coordinación entre profesores y estudiantes del master para continuar con la docencia no presencial; un sinfín de tutorías ad hoc a través de correos electrónicos y del aula virtual; cumplimentar las fichas del sistema de evaluación alternativo para las todas las asignaturas que coordino, que son una de grado y cuatro de master (esto ya una vez decretado el plan de contingencia por parte de la UMU); reuniones virtuales y telefónicas con otros profesores y equipo decanal de la Facultad de Medicina; y seguramente, más cosas que no se encuadran en las anteriores, sin límites de días u horarios, pero han servido para avanzar manteniendo el necesario rigor académico con la flexibilidad que esta situación excepcional requiere.

Con excepción de las Prácticas Clínicas, las asignaturas que coordino e imparto están en parte virtualizadas. Además, ya se habían impartido contenidos de forma presencial antes de marzo, y no he tenido que utilizar el aula virtual para seguir con el desarrollo de las clases en el horario habitual. A través del aula virtual, en la sección de recursos, se ha dotado a los estudiantes de material de estudio, adecuando el plan de trabajo presencial a las herramientas online.

Para la presentación de los trabajos escritos realizados grupalmente, los estudiantes de Máster han aportado en el aula virtual tanto el trabajo escrito como la presentación, y la corrección de los mismos, en lugar de en la Facultad, se efectúa en el domicilio de la profesora, y el feedback es en diferido en lugar de inmediato. Así mismo, los trabajos individuales, consistentes en emitir los informes resultantes de administrar a dos casos reales instrumentos de evaluación de marcha y equilibrio y de discapacidad, han sido entregados por los estudiantes a través del aula virtual o por correo electrónico. Sobre la realización de estos dos casos, tenía previsto modificar ese aspecto de la evaluación en la ficha de contingencias de evaluación, y solicitar la evaluación en supuestos prácticos y no en casos reales (por la dificultad derivada de la la prohibición de contacto), pero todos los estudiantes han presentado los trabajos en la forma prevista (hay que tener en cuenta que podían ser pacientes o personas mayores de su entorno).

Respecto a las tutorías, la mayor parte de las veces, la respuesta ha sido inmediata, pero, en cualquier caso, el tiempo de respuesta nunca ha excedido las 48 horas (lectivas o en días festivos). Cuando una consulta ha requerido información grupal, además de la respuesta individual, he informado grupalmente mediante un anuncio en la plataforma virtual dirigida a todos los participantes.

En cuanto a los criterios de evaluación, a diferencia de la presencialidad prevista para los exámenes escritos, en las fichas de contingencias he incorporado que se realizarán a través del aula virtual. 


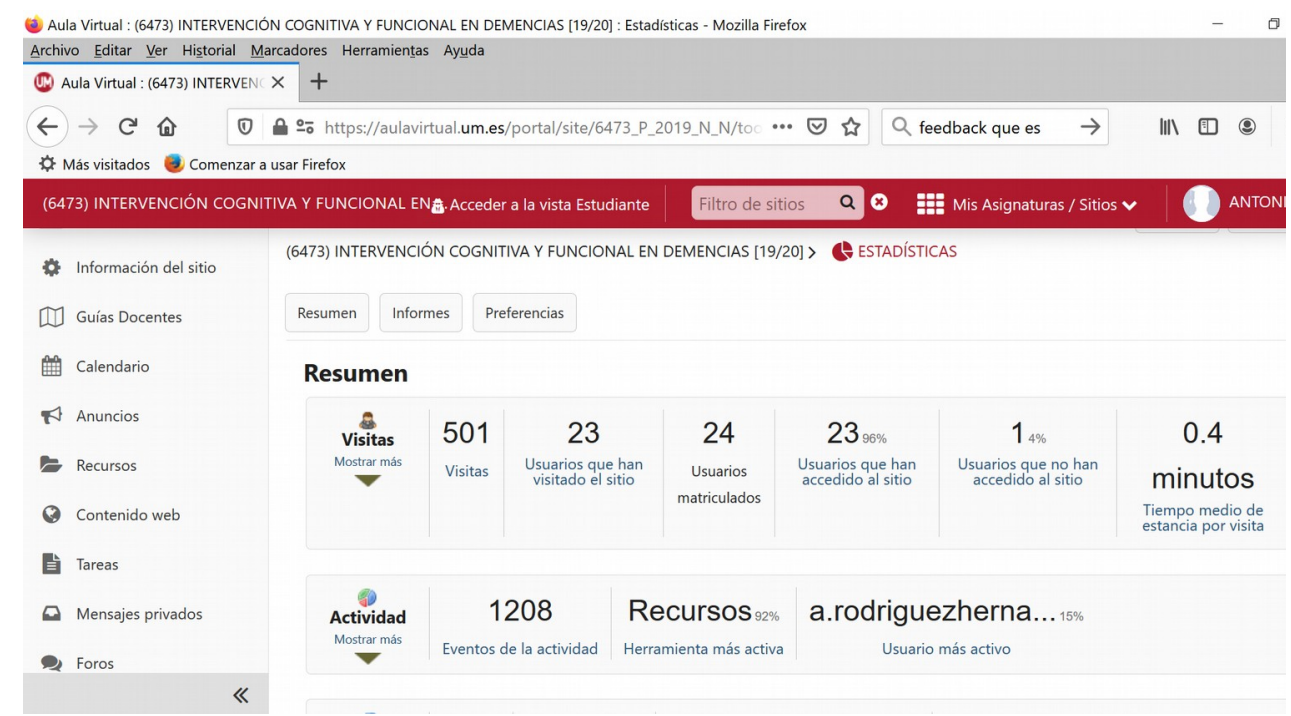

Figura 1. Resumen de las actividades de la asignatura de máster

No se dispone de datos sobre la satisfacción de los estudiantes, pero dado los múltiples agradecimientos que me han enviado cada vez que he llevado a cabo alguna de las acciones descritas previamente, mi percepción es que se han sentido atendidos, y he podido adaptar la docencia y la organización, y transmitirles cierta tranquilidad para el avance de las asignaturas, y en el caso del master, del curso y la titulación.

Las figuras 1 a 4, son ejemplos de las actividades y visitas en el último mes en el aula virtual correspondiente a dos asignaturas, una de grado y la otra de máster.

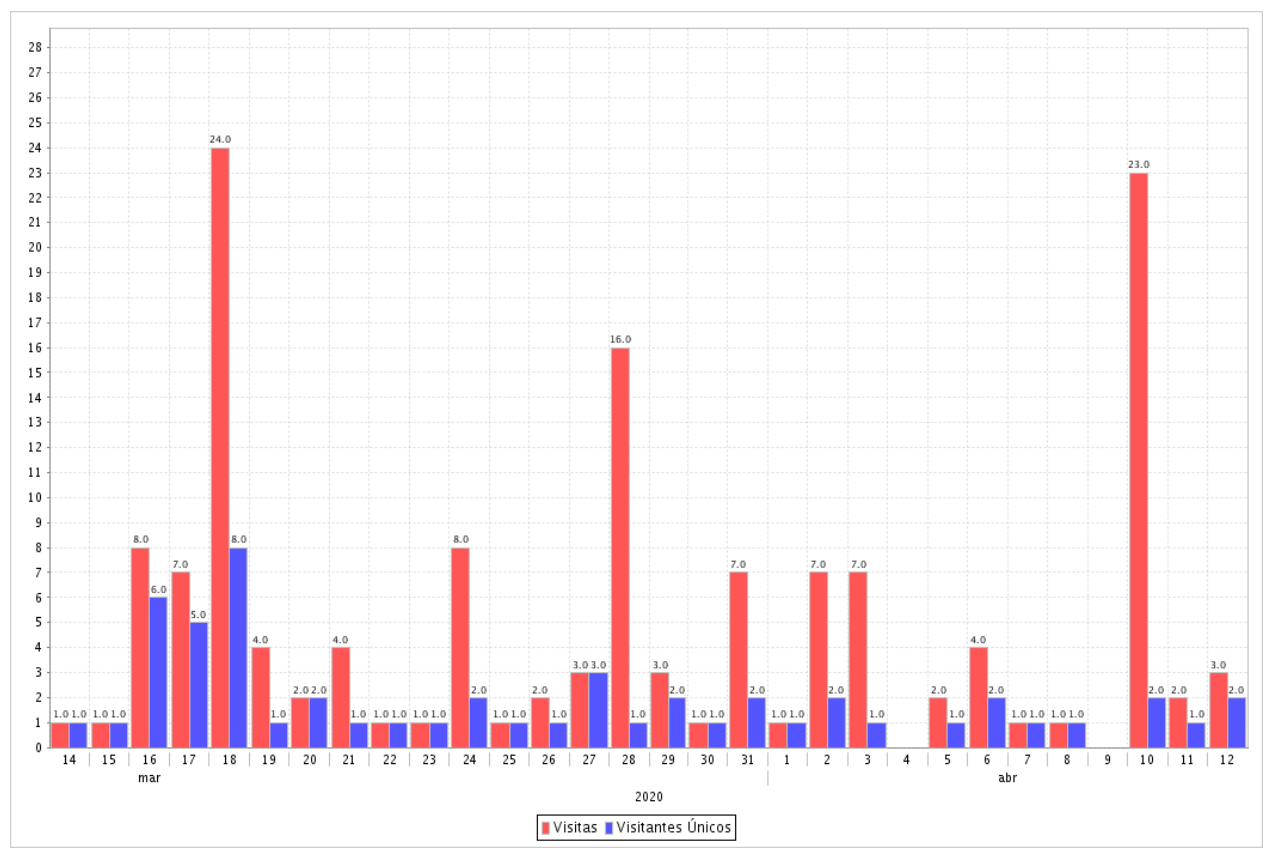

Figura 2. Visitas en los últimos 30 días en la asignatura del máster 


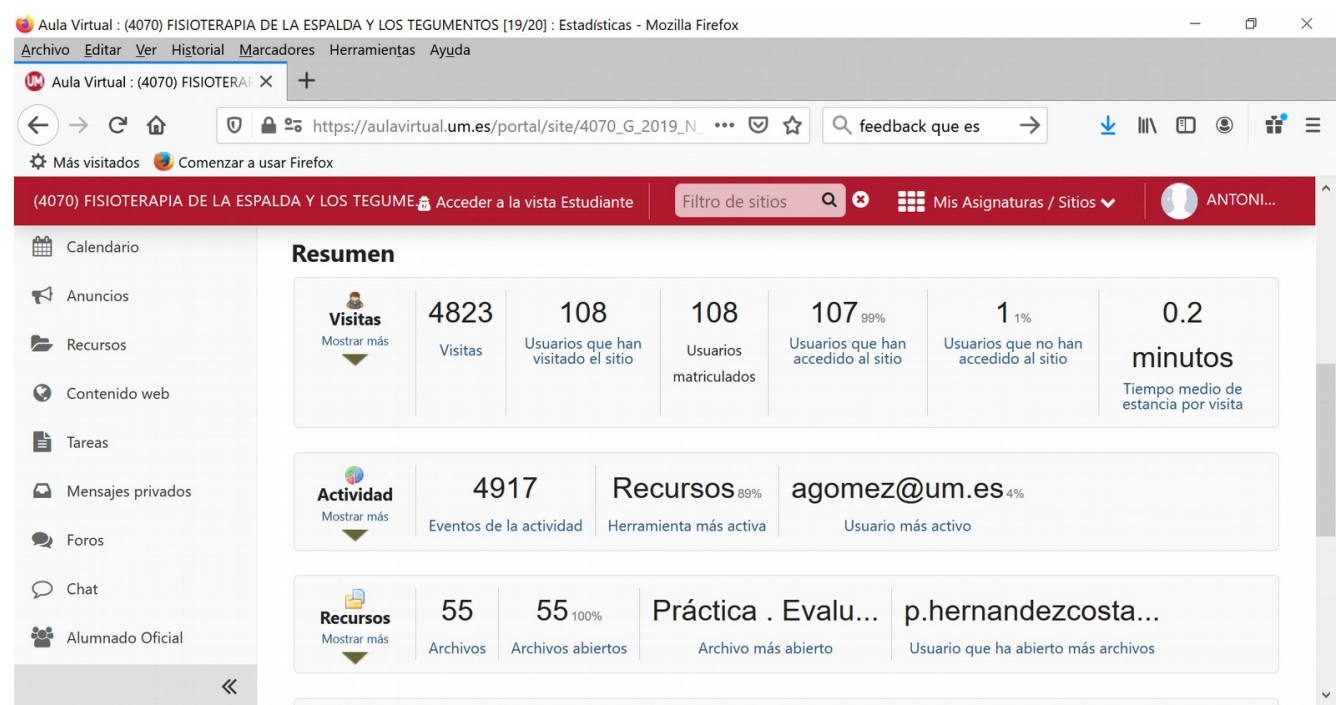

Figura 3. Resumen de las actividades en la asignatura del grado (3ํㅡㄴ curso)

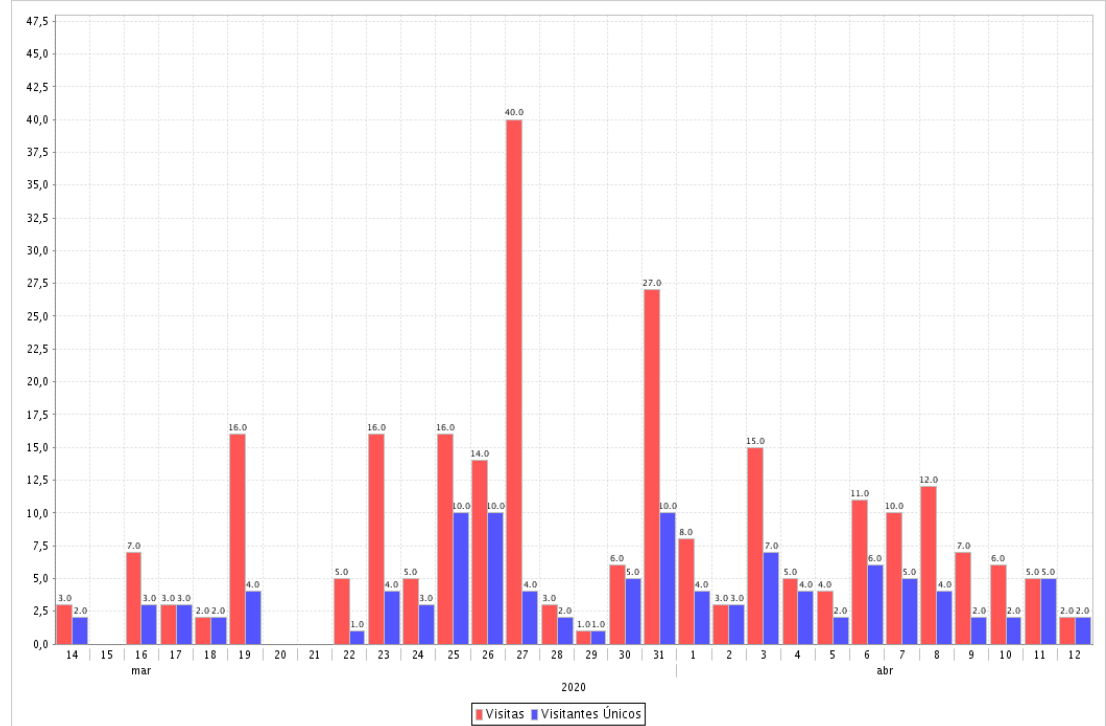

Figura 4. Visitas en los últimos 30 días en la asignatura de grado ( $3^{\circ}$ curso)

\section{Clínica Integrada de Infantil II (COII-II), Grado en Odontología, Prof. Antonio J. Ortiz Ruiz.}

La asignatura Clínica Integrada de Infantil II (COII-II) forma parte del Practicum del Grado de Odontología. Pertenece al segundo cuatrimestre de quinto curso y tiene una carga de 6 ECTS. La metodología docente utilizada es la realización de tres seminarios temáticos donde se resuelven 9 casos clínicos con exposición de los trabajos por parte del alumnado, y el resto es práctica clínica con pacientes infantiles. Previamente a cada práctica clínica el alumno presenta el plan de tratamiento para su discusión en sesiones clínicas de 1 hora de duración.

El plan de contingencia propuesto para la asignatura COII-II para el periodo de suspensión de la actividad docente presencial fue la realización de seminarios y sesiones clínicas a través del aula virtual. Se han colgado casos clínicos reales, los alumnos los han resuelto, realizando búsquedas bibliográficas actualizadas en las bases de datos científicas, y han subido al aula virtual un documento que contiene las respuestas a las preguntas que les hemos propuesto como guía para la resolución, que son el diagnóstico, la justificación del diagnóstico, el diagnóstico diferencial, el plan de tratamiento, los tratamientos (procedimientos, materiales), los tratamientos alternativos, la justificación del tratamiento, las referencias bibliográficas utilizadas para resolver el caso. 
El cambio en la metodología docente se ha visto reflejado en el uso de la asignatura del aula virtual. En la tabla 1 se puede observar la comparación entre la actividad del aula virtual en el curso anterior y en el actual. De las actividades, 823 son descargas a recursos y 425 mensajes privados.

\begin{tabular}{|c|c|c|c|c|c|}
\hline COII II & & \multicolumn{2}{|c|}{ Total } & \multicolumn{2}{c|}{ Estudiante } \\
\hline & & Visitas & Actividades & Visitas & Actividades \\
\hline $\mathbf{2 0 1 8 - 1 9}$ & Febrero & 58 & 75 & 54 & 72 \\
\hline \multicolumn{7}{|c|}{ Marzo } & 26 & 51 & 23 & 45 \\
\hline $\mathbf{2 0 1 9 - 2 0}$ & Febrero & 41 & 58 & 33 & 51 \\
\hline & Marzo & 1459 & 1767 & 1369 & 1302 \\
\hline
\end{tabular}

Tabla 1: actividad del sitio de la asignatura COII-II en los cursos 2018-19 y 2019-20.

En las asignaturas del practicum se engloban las prácticas preprofesionales que, en el caso de los grados en Odontología, se desarrollan en las Clínicas Odontológicas de las Universidades. En la Universidad de Murcia, la Clínica Odontológica está ubicada en el Hospital Universitario Morales Meseguer. Desde la anulación de las actividades presenciales de los alumnos a partir del 16 de marzo de 2020, por resolución del Rector de 13-03-2020, las prácticas clínicas quedaron suspendidas.

Sustituir una práctica clínica por una actividad online es imposible, ya que las habilidades y las actitudes frente al paciente, el caso clínico real, solo se consiguen con el paciente delante. Hemos optado, como alternativa a las prácticas clínicas, por la resolución de casos clínicos reales usando la herramienta "videoconferencia" del aula virtual, donde el alumno hace la presentación del caso clínico resuelto según la guía antes presentada con la participación simultánea de todos los alumnos y profesores en la discusión. Todos los alumnos deben de subir al aula virtual los casos resueltos antes del inicio de la clase. Para conocer lo que los alumnos piensan sobre el cambio de metodología hemos desarrollado una pequeña encuesta. De los 38 alumnos matriculados han contestado 22. La preguntas y resultados son los siguientes:

- $\quad \dot{T}$ e ha parecido positiva la experiencia del uso de la herramienta "videoconferencia" del aula virtual para desarrollar los seminarios de la asignatura? Sí $=22 ; \mathrm{No}=0$

- ¿ ¿Te ha resultado fácil el manejo de la aplicación? $\mathrm{Si}=22 ; \mathrm{No}=0$

- ¿Crees que puedes participar mejor a través de la videoconferencia que en modo presencial? Sí=5; No=16

- ¿ ¿Crees que resta calidad a tu formación este tipo de metodología docente para la realización de los seminarios y resolución de casos clínicos? Sí=1; No=21

- ¿ ¿Preferirías que en el futuro la asignatura COII usara la videoconferencia para la realización de los seminarios? $\mathrm{Si}=14 ; \mathrm{No}=8$.

La evaluación de la asignatura viene recogida en la guía docente inicial de la siguiente forma: un examen teórico de todo los contenidos de la asignatura $(60 \%)$, exposición y presentación de los casos clínicos (10\%) y presentación de los casos clínicos (30\%). En el plan de continencia 2 se recoge la nueva evaluación de la asignatura que queda así: se valorará la exposición y la presentación de los seminarios $(60 \%)$ y los casos clínicos (10\%) realizados tanto presencialmente, antes del confinamiento, como a través de videoconferencia; la práctica clínica (30\%) se realizará con evaluación continua, siguiendo los criterios publicados en la guía docente, de las 6 prácticas clínicas (el $62.5 \%$ del total) ya realizadas hasta el momento de la suspensión de las clases presenciales. 


\section{Patología Quirúrgica I y Fundamentos de Cirugía, Grado en Odontología y Grado en Medicina, Prof. Pedro Cascales Campos.}

En estas asignaturas, de $2^{\circ}$ curso del grado de Odontología (Patología Quirúrgica I) y de 3o curso del Grado en Medicina (Fundamentos de Cirugía), aproximadamente el $75 \%$ de la teoría estaba finalizada al inicio del estado de alarma. En estas circunstancias excepcionales, se ha mantenido el contenido de la asignatura y se ha adaptado en forma de un material online, que se ha ido subiendo al aula virtual de la UMU, en formatos powerpoint, pdf y acompañados de vídeos explicativos. La realización del trabajo autónomo por parte del alumno no se ha visto alterada ya que, desde el primer momento, se planteó desde el aula virtual. En el caso del grado de Odontología, el trabajo autónomo se centraba en describir un hito en la historia de la odontología, focalizado en la práctica quirúrgica y que hubiera impactado en el quehacer actual de los Odontólogos.

Una de las inquietudes que más afecta a los estudiantes es la forma de evaluar que el profesorado va a utilizar en estas asignaturas del cuatrimestre afectadas por el estado de alarma. En este sentido, mantendremos el formato clásico y reflejado en la guía docente de evaluación mediante pruebas tipo test que se realizarán utilizando la herramienta exámenes del aula virtual. Al igual que los estudiantes, el profesorado también tiene que pasar por un periodo de adaptación y tendrá que mantener una postura cautelosa respecto al nivel de exigencia en la evaluación, fundamentalmente. Esto supondrá, sin duda, un esfuerzo adicional para intentar salvaguardar la igualdad de condiciones y oportunidades, individualizando cada caso particular del alumno.

Finalmente, debemos hacer una reflexión. El principal problema docente de las asignaturas clínicas reside en adaptar la metodología de la enseñanza práctica en el hospital. La imposibilidad de poder contar físicamente con los alumnos en el hospital y en las salas de simulación, dificultad este hecho. Herramientas como www.medicalum.com pueden ser útiles en este sentido ya que cuentan con un extenso material clínico que, si bien tiene las limitaciones evidentes de no poder estar presencialmente en la cabecera del paciente, puede generar una estructura de aprendizaje basada en la resolución de problemas habituales de la práctica clínica diaria los alumnos. Estos cambios que ahora se han planteado, deben ser un acicate para intentar mejorar algunas cosas que no funcionan bien en la docencia universitaria. Adaptar a la docencia una frase que habitualmente decimos en cirugía es pertinente ahora: "El buen cirujano (docente) aprende de sus errores; el mejor cirujano (docente) aprende también de los errores de los demás; hay cirujanos (docentes) que nunca aprenden". No seamos como los del último ejemplo. Lo que se ha hecho antes del estado de alarma, ya es el pasado.

\section{Fisiología I, Grado en Medicina, Prof. Joaquín García-Estañ López.}

En el grupo 2 de Fisiología I se ha completado la planificación prevista inicialmente mediante el uso de la Videoconferencia y del Chat del aula virtual. Las clases, además, se han grabado mediante la aplicación de videoconferencia del aula virtual y están a disposición de todos los estudiantes en la herramienta Videoapuntes del aula virtual.

El comienzo del período de confinamiento prácticamente coincidió con la última sesión de prácticas presencial (viernes 13 de marzo) a la que ya sólo vinieron 3 estudiantes (de unos 20 que componen el grupo). Eran prácticas de Electrocardiografía y en esta sesión, con la ayuda de estos tres estudiantes, se grabaron vídeos de la explicación y realización de la práctica. Para ello, usamos nuestros propios teléfonos móviles. Estos vídeos se alojaron posteriormente en 
YouTube, en la cuenta personal del profesor, aunque posteriormente se han colocado en la herramienta Videoapuntes del aula virtual, compartiéndolos con toda la clase a través de un anuncio en el sitio de la asignatura.

También se ha realizado, de manera online gracias a la herramienta Exámenes del aula virtual, el examen parcial previsto (26 de marzo), a plena satisfacción. Se presentaron 86 estudiantes y los resultados han sido muy similares a los de los parciales de los cursos anteriores. El examen constó de 30 preguntas tipo test (4 respuestas posibles) y duró 40 minutos exactos. Creo que, a expensas de lo que la Universidad dictamine, se puede evaluar a los estudiantes de esta manera, salvo que volvamos a las aulas, lógicamente.

Finalmente se ha realizado, también a través de videoconferencia, un seminario (1 de abril) como estaba previsto. Se conectaron y participaron unos 60 estudiantes, del total de 100 matriculados. La actividad consistió en resolver problemas de Fisiología Cardiovascular enviados previamente a los estudiantes en forma de documento de texto con espacio para rellenar. Durante el seminario, se iba preguntando mediante audio a los estudiantes la solución a cada pregunta y cuando había consenso entre ellos, la respuesta se anotaba en el documento del profesor, compartido en la pantalla de la videoconferencia. Comentemos que los estudiantes no usaron el audio de la videoconferencia ni compartieon su vídeo con lo que tampoco se les puede ver, lo que es un poco desconcertante. Por lo tanto, toda la comunicación por parte de ellos es escrita en el chat de la videoconferencia.

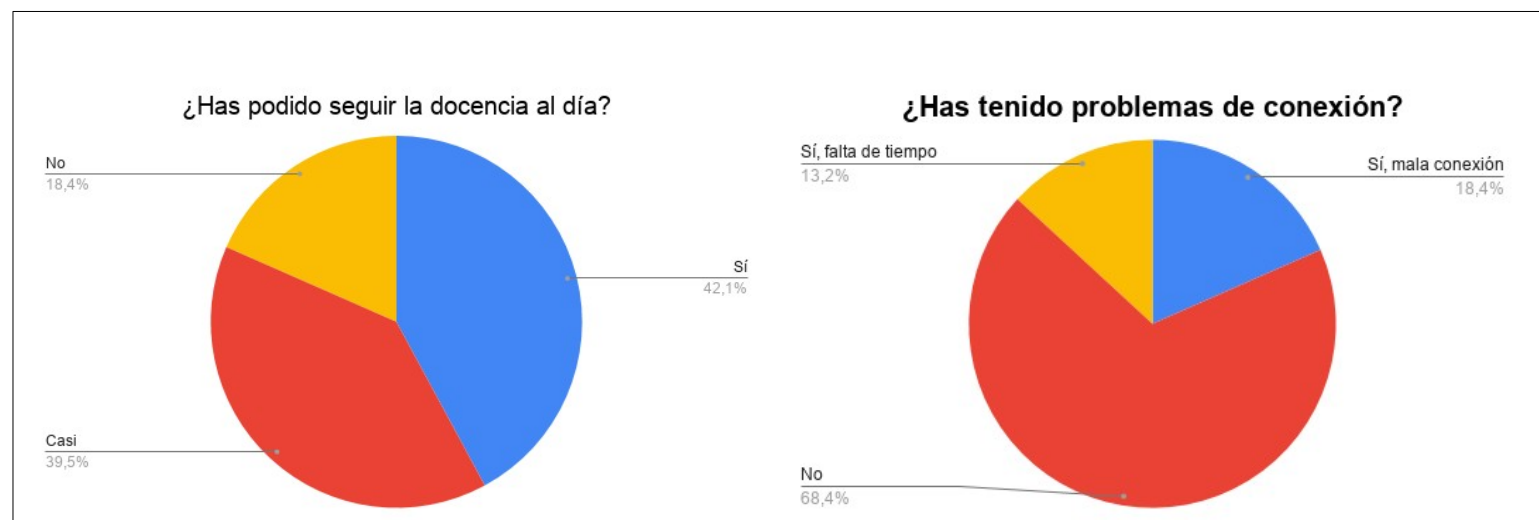

Figura 5. Seguimiento de la docencia virtual

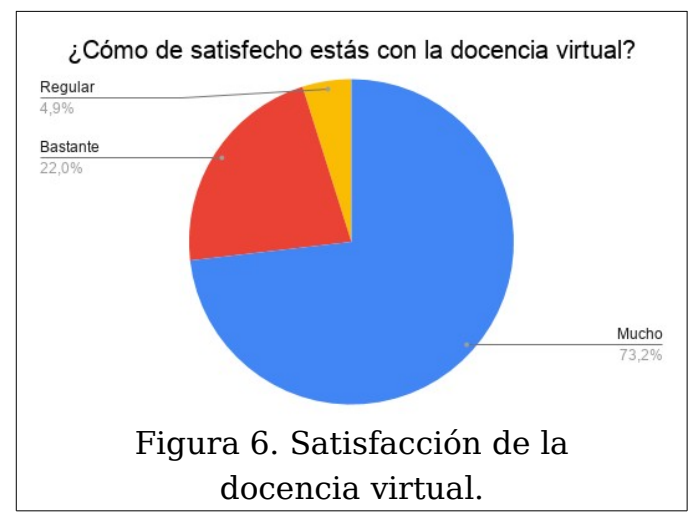

El último día de docencia del cuatrimestre (3 de abril) se distribuyó una encuesta después de la clase a través de Google Forms, contestando 41 estudiantes. En la figura 5 se muestran las respuestas sobre el seguimiento de la docencia o los problemas que algunos comentaban en sus mensajes. Sobre la satisfacción, la mayoría contestaron estar bastante o muy satisfechos (figura 6). 
El resto del temario y demás actividades, después del período de semana santa y fiestas de primavera, se prevé realizarlo de igual manera, grabación de las clases y realización de las prácticas (a través del uso de escritorio virtual) con software de simulación. Desafortunadamente, las prácticas de hematología que son de laboratorio no se podrán hacer, pero la simulación debe proporcionar una alternativa razonable.

(C) 2020 por los autores. Enviado para su publicación en acceso abierto bajo los términos y condiciones de la licencia Creative Commons Attribution (CC BY) (http://creativecommons.org/licenses/by/4.0/). 